\title{
Establishment of an insufficient radiofrequency ablation orthotopic nude mouse model of hepatocellular carcinoma to study the invasiveness and metastatic potential of residual cancer
}

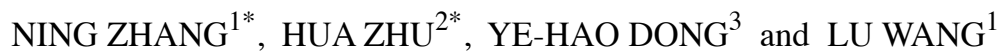 \\ ${ }^{1}$ Department of Hepatic Surgery, Fudan University Shanghai Cancer Center, Shanghai 200032; \\ ${ }^{2}$ Department of Oncology, The First People's Hospital of Jining; ${ }^{3}$ Reproductive Medicine Center, \\ The Affiliated Hospital of Jining Medical College, Jining, Shandong 272000, P.R. China
}

Received May 31, 2018; Accepted February 19, 2019

DOI: $10.3892 / 01.2019 .10552$

\begin{abstract}
In order to assess the metastatic potential of residual hepatocellular carcinoma (HCC) following insufficient radiofrequency ablation (RFA) and to improve the current animal model, an insufficient RFA orthotopic nude mouse model of HCC was developed in the present study. A human HCC orthotopic nude mouse model was established using HCCLM3 cells, which has a high metastatic potential, labeled with green fluorescent protein. A total of 12 nude mice within the RFA group received insufficient RFA and 12 mice in the control group received RFA needle electrode puncture of the tumor without ablation, 3 weeks after implantation. To investigate tumor growth and metastasis, 4 weeks after RFA, six mice in each group were sacrificed and the remaining mice in each group were maintained until death to evaluate their life span. No mice died following insufficient RFA and the success rate was $100 \%$. Compared with the control group, the intrahepatic and lung metastasis rates were higher in the RFA group, despite the mice having smaller tumor volumes and longer survival times. Lung and intrahepatic metastasis rates in the insufficient RFA group were 100\% (6/6) and 66.67\% (4/6), respectively, compared with $33.33 \%$ (2/6) and $0 \%(0 / 6)$, respectively, in the control group. As part of the study, a safe and reliable method to establish an insufficient RFA orthotopic nude mouse model was developed. The present study revealed that residual cancer following insufficient RFA had exhibited increased invasiveness and metastatic potential.
\end{abstract}

Correspondence to: Professor Lu Wang, Department of Hepatic Surgery, Fudan University Shanghai Cancer Center, 270 Dong An Road, Shanghai 200032, P.R. China

E-mail:cms024mm@163.com

${ }^{*}$ Contributed equally

Key words: hepatocellular carcinoma, residual cancer, insufficient radiofrequency ablation, nude mouse, orthotopic transplantation tumor

\section{Introduction}

Primary liver cancer is the fifth most common type of cancer and the third leading cause of cancer-related mortality worldwide, with hepatocellular carcinoma (HCC) accounting for $>90 \%$ of cases (1). Although surgical resection is the standard treatment modality for HCC, its use is limited, as the majority of patients, even in those with small-sized tumors, also have associated severe liver dysfunction $(2,3)$. Liver transplantation provides an alternative curative treatment for small unresectable HCC in patients with small-sized tumors. However, the shortage of liver grafts limits the applicability of this approach (4).

Radiofrequency ablation (RFA) is a previously developed technique for ablation of liver tumors and is well tolerated in patients with unresectable HCC (5). In 2012, a randomized controlled trial also revealed that percutaneous RFA was as effective as hepatic resection in terms of overall survival and recurrence-free survival times in the treatment of patients with small HCC tumors $(<4 \mathrm{~cm})(6)$. Despite its technical simplicity and safety, the recurrence rate following RFA remains high (48-95\%) (7-9) as the complete ablation of the tumor is difficult to achieve (9). To investigate the biological characteristics of residual cancer following RFA, a model of insufficient RFA is important.

In the present study, an orthotopic nude mouse model of HCC was developed to assess the metastatic potential of residual cancer following insufficient RFA and to improve the current animal model.

\section{Materials and methods}

Cell line. The green fluorescent protein (GFP)-expressing HCCLM3 cell line (HCCLM3-GFP), derived from HCCLM3 cells (established by the Liver Cancer Institute of Fudan University, Shanghai, China) (10), was used for in vivo experiments. The cells were maintained in Dulbecco's modified Eagle's medium with $10 \%$ fetal bovine serum and $100 \mathrm{mg} / \mathrm{ml}$ penicillin G (all from Thermo Fisher Scientific, Inc., Waltham, MA, USA) at $37^{\circ} \mathrm{C}$ in a humidified atmosphere containing $5 \% \mathrm{CO}_{2}$. 
Animal model. A total of 24 male athymic BALB/c nu/nu mice, weighing 18-20 g at 4-6 weeks of age, were obtained from the Shanghai Laboratory Animal Centre Co., Ltd. (Shanghai, China). All mice were housed in specific pathogen-free conditions (temperature, $26-28^{\circ} \mathrm{C}$; atmosphere, $0.65 \mathrm{~cm} \mathrm{H}_{2} \mathrm{O} ; 10 / 14 \mathrm{~h}$ light/dark cycle; with ad libitum access to food and water). All animal protocols were approved by the Ethical Committee on Animal Experiments of the Animal Care Committee of Fudan University (Shanghai, China). All efforts were made to minimize animal suffering.

Equipment. The main equipment comprised a $1500 \mathrm{X}$ RF Generator (RITA Medical Systems, Inc.; AngioDynamics, Inc., Latham, NY, USA), retractable multiple hook RFA needles (Fig. 1; RITA Medical Systems, Inc.; AngioDynamics, Inc.), and RITA grounding pads (RITA Medical Systems, Inc.; AngioDynamics, Inc.).

Establishment of an orthotopic transplantation tumor model of HCC in nude mice. The HCCLM3-GFP cells $\left(1 \times 10^{7}\right)$ were subcutaneously inoculated into the right flanks of two 4-week-old BALB/c nu/nu male mice. Subcutaneous ectopic tumors were harvested upon reaching $\sim 1 \mathrm{~cm}$ in diameter, after 3-4 weeks. Prior to subcutaneous ectopic tumor removal, general anesthesia was induced with $50 \mathrm{mg} / \mathrm{kg}$ pelltobarbitalum natricum (Haoran Biological Technology Co., Ltd., Shanghai, China) administered by intraperitoneal injection. Following the removal of the necrotic tissues, the tumors were cut into $\sim 1-\mathrm{mm}^{3}$ sections under aseptic conditions. Following anesthesia with pelltobarbitalum natricum, at the aforementioned dose, the abdominal cavity of the mice was opened and the left liver lobe was made accessible. Ophthalmic ligature forceps were used to create a small tunnel under the surface of the left liver lobe and the trimmed tumor was placed into the tunnel. Finally, the opening of the tunnel was closed by a 6-0 non-absorbable suture. The nude mice bearing xenografts were randomly divided into a sham-operated group $(n=12)$ and an insufficient RFA group $(n=12)$ when the orthotopic transplantation tumor had reached $\sim 0.5 \mathrm{~cm}$ in diameter, $\sim 3$ weeks after implantation.

Insufficient RFA. Insufficient RFA was performed 3 weeks after tumor implantation. Following anesthesia, the abdomen was prepared with iodine and alcohol scrub, and draped with sterile towels. Initially, the mouse was placed on a conductive metal plate and its limbs were fixed. RITA grounding pads were adhered to the back of the metal plate. Good electrical conductivity was maintained between the metal plate and the back of the nude mouse. The abdominal cavity of the mouse was opened to expose the left liver lobe and the transplanted tumor. During implantation, the graft was embedded under the capsule of the left lobe of the liver, such that after 3 weeks, the protruding transplanted tumors were visible with the naked eye on the surface of the liver. The center straight needle of the RF probe with an active diameter of $4 \mathrm{~mm}$ was inserted into the tumor, and normal saline was dripped into the puncture site to maintain good conductivity. An active diameter of $4 \mathrm{~mm}$ for the probe was determined to be optimal based on preliminary experiments (data not shown). Taking into account the weight and volume of the tumor, RFA was performed using a low-energy protocol, in which the output power was $5 \mathrm{~W}$ and the duration was $30 \mathrm{sec}$. Following RFA, the abdominal cavity of the mice was closed using 5-0 non-absorbable sutures. The control group underwent a sham surgery by inserting a needle electrode into the tumor without performing RFA.

Tumor volume and survival time of nude mice. To evaluate tumor growth and metastasis, 6 nude mice from the 2 aforementioned groups were sacrificed 4 weeks after either insufficient RFA treatment or a sham operation. Tumors were excised and their largest (a) and shortest (b) diameters were measured to calculate tumor volume as follows: Tumor volume $=\mathrm{a} x$ $\mathrm{b}^{2} / 2$ (11). To evaluate the survival time, the remaining mice in each group were maintained until death.

Lung metastasis and intrahepatic metastasis (IHM) evaluation. To evaluate the metastatic potential of residual tumor following insufficient RFA, the area of green fluorescence, which indicated the lung metastatic nodes, was measured. The images of GFP-positive metastatic foci were captured by stereoscopic fluorescence microscopy (magnification, x10; Leica Microsystems GmbH, Wetzlar, Germany). The lung tissues were sectioned $(4 \mu \mathrm{m})$ serially, and hematoxylin and eosin staining (room temperature; hematoxylin, $10 \mathrm{~min}$; eosin, $30 \mathrm{sec}$ ) confirmed the aforementioned results. IHM was observed by fluorescent imaging and quantified as number per liver.

Statistical analysis. Data were analyzed using SPSS 22.0 (IBM Corp., Armonk, NY, USA). The results are expressed as the mean \pm standard deviation. Statistical analysis was performed using Student's t-test. The Kaplan-Meier method with log-rank test was used for survival analysis. $\mathrm{P}<0.05$ was considered to indicate a statistically significant difference.

\section{Results}

Establishment of insufficient RFA orthotopic nude mouse model of HCC. No nude mice died following insufficient RFA. If ablation of necrotic tissue and residual tumor was clearly observed under fluorescence microscopy, it revealed that insufficient RFA was successful (Fig. 2), and the success rate in the insufficient RFA group was $100 \%$. In the control group, all the nude mice were alive until the evaluation of metastasis.

Insufficient RFA reduces tumor volume and prolongs survival time in nude mice. The tumor volume of the HCCLM3-GFP-derived xenografts was $449.58 \pm 350.75 \mathrm{~mm}^{3}$ in the insufficient RFA group, which was significantly smaller compared with that of the matched sham-operated controls (1788.66 $\pm 608.80 \mathrm{~mm}^{3}$; $\mathrm{P}<0.05$; Fig. 3A). The mean survival time in the insufficient RFA group was significantly longer compared with that in the sham-operated control group (80.8 \pm 3.5 days vs. $75.0 \pm 3.3$ days; $\mathrm{P}<0.05$; Fig. $3 \mathrm{~B}$ ).

Insufficient RFA promotes invasiveness and distant metastasis. Green fluorescence imaging revealed that insufficient RFA promoted intrahepatic dissemination (Fig. 4A). The IHM rate in the insufficient RFA group was $66.67 \%(4 / 6)$, but no IHM was observed in the control group $(\mathrm{P}<0.05)$. 


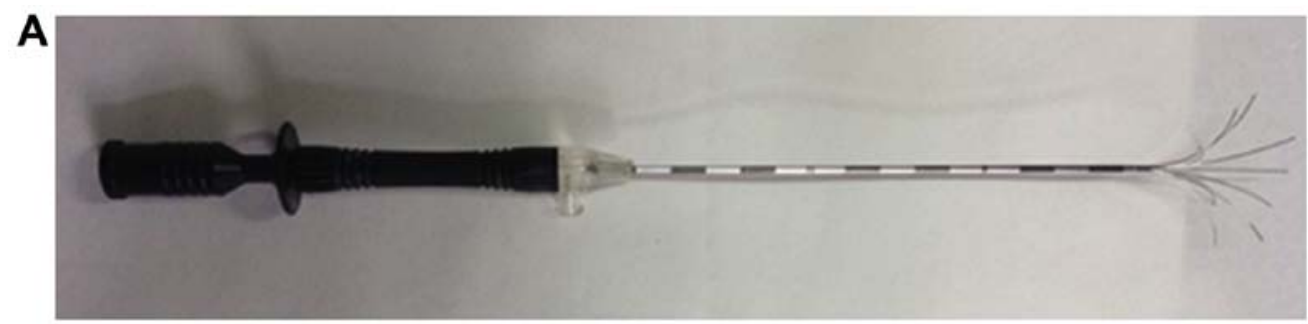

B

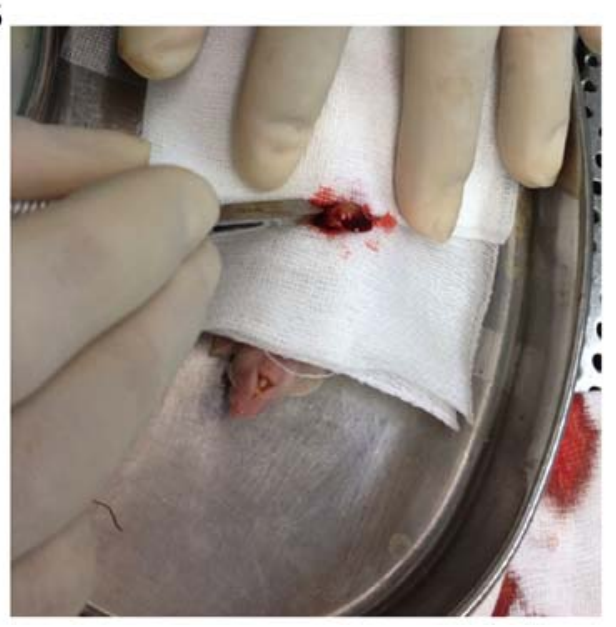

C

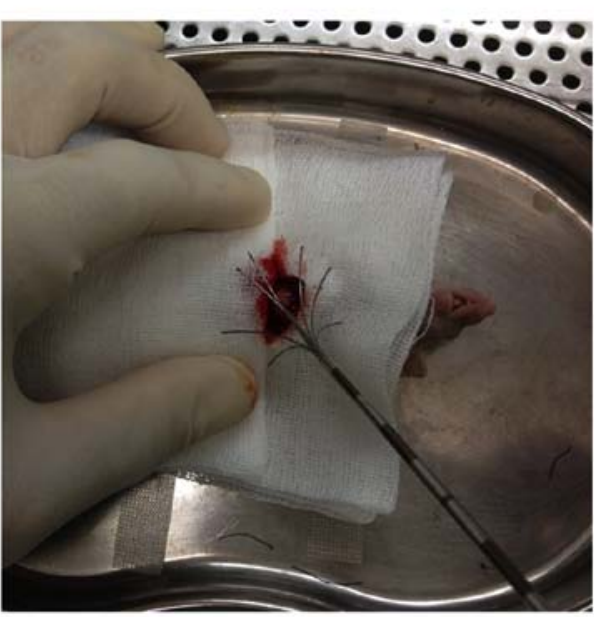

Figure 1. Equipment for insufficient RFA in an orthotopic nude mouse model of HCC. (A) A retractable multiple hook RFA needle. (B) Opening the abdominal cavity and fully exposed xenograft tumor with the left liver lobe. (C) A retractable RFA needle was extended and the middle straight thin needle was inserted into the xenograft tumor. RFA, radiofrequency ablation.
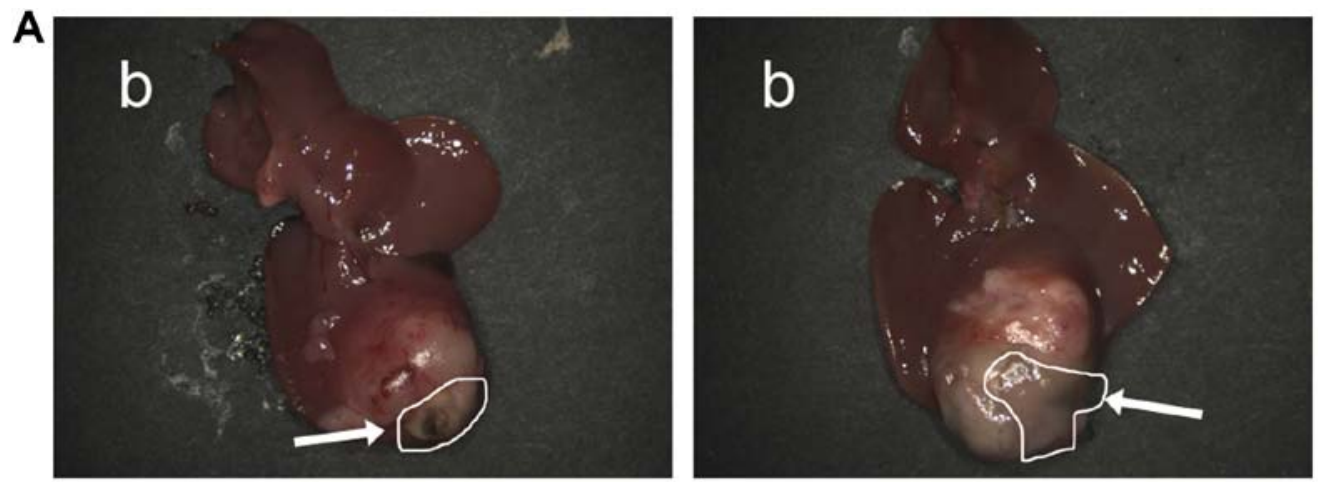

B
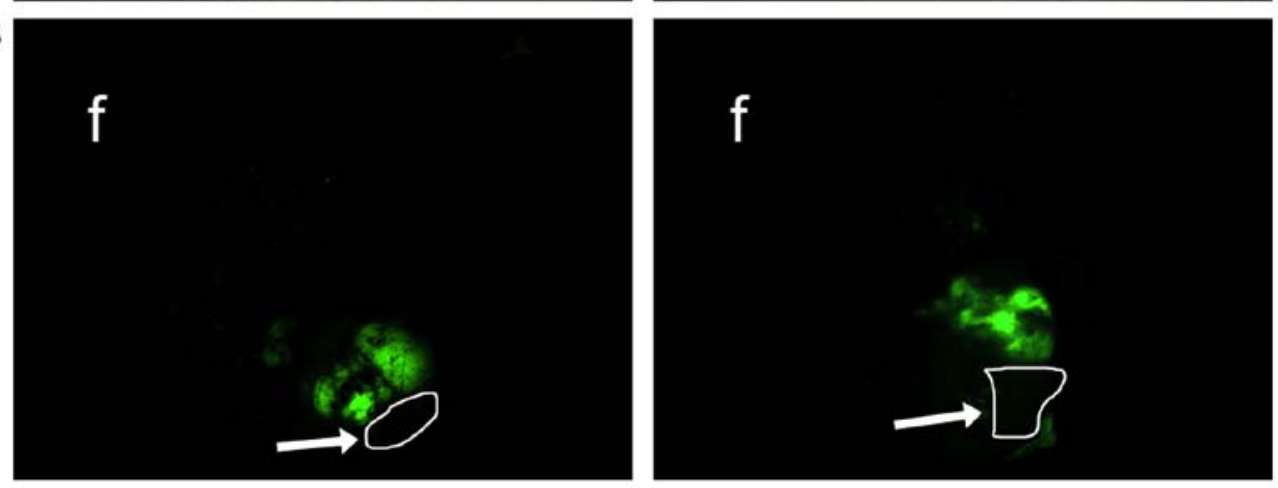

Figure 2. Effect of insufficient RFA on orthotopic tumors. (A) Gray area (white arrow) represents the necrotic tissue following insufficient RFA. Tissue surrounding the gray area is the residual tumor. (B) Xenograft tumor from (A) observed under fluorescence microscopy. The green fluorescent area indicates the residual tumor. The black area (white arrow) between two green fluorescent areas indicates necrotic tissue. RFA, radiofrequency ablation; b, bright field; f, fluorescence.

The lung metastasis rate in the insufficient RFA group was $100 \%$ (6/6), compared with $33.33 \%(2 / 6)$ in the control group $(\mathrm{P}<0.05$; Fig. 4B). Analysis of serial lung paraffin sections indicated a significantly higher incidence of lung metastasis in the insufficient RFA-treated mice compared with that in the sham-operated mice (Fig. 4C). 

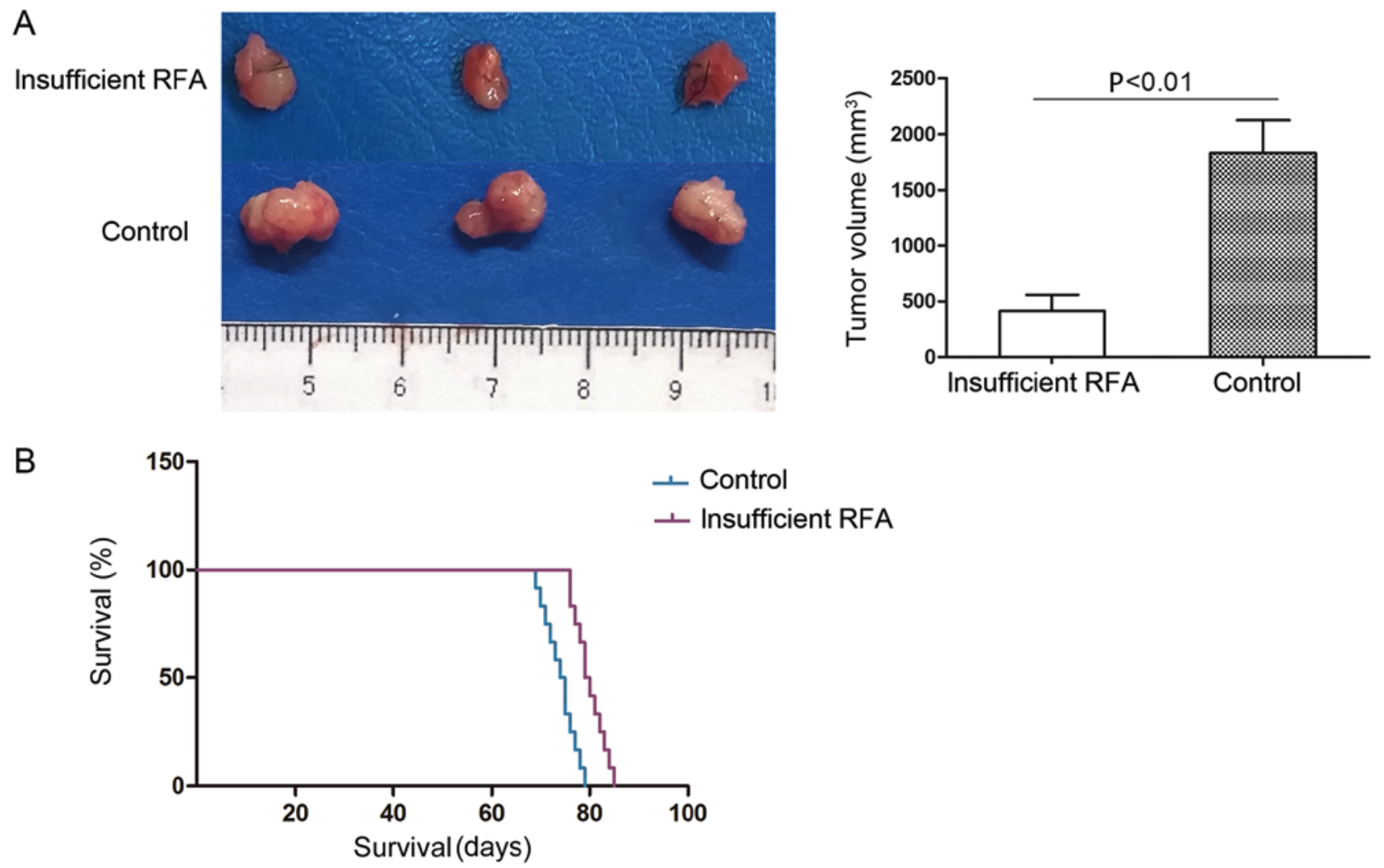

Figure 3. Insufficient RFA reduces tumor volume and prolongs survival time in nude mice. (A) Xenograft tumor volume was decreased in the insufficient RFA group compared with that in the control group. Tumor volume was $1788.66 \pm 608.80 \mathrm{~mm}^{3}$ in the control group and $449.58 \pm 350.75 \mathrm{~mm}^{3}$ in the insufficient RFA group. (B) Insufficient RFA prolonged the survival time of the tumor-bearing nude mice; the survival time in the control group was $75.0 \pm 3.3$ days compared with $80.8 \pm 3.5$ days $(\mathrm{P}<0.05)$ in the insufficient RFA group. RFA, radiofrequency ablation.

\section{Discussion}

To investigate the invasiveness and metastatic potential of a residual tumor following insufficient RFA, an appropriate orthotopic animal model is required. The majority of research utilizes the nude mice model and its use in investigating a variety of HCC therapies is not novel. The orthotopic nude mouse model of HCC was initially described in 1996 (12) and the orthotopic mouse model has served as an ideal experimental tool to investigate $\mathrm{HCC}$ and its treatment since 1998 (13). However, there are few reports regarding the nude mouse model in RFA research. To date, the majority of in vivo studies investigating the biological behavior of RFA of tumors have used nude mouse subcutaneous xenograft models or a rabbit orthotopic model (14-17). Xu et al (15) inoculated HCC cells subcutaneously into the right flank of mice. After 14 days, the subcutaneous xenograft tumor was treated with a partial RFA strategy using $180 \mathrm{sec}$ of RFA at $1 \mathrm{~W}$ once the tumor length had reached $12-15 \mathrm{~mm}$. There are no reports regarding the biological characteristics of residual HCC following RFA using an orthotopic nude mouse model, to the best of our knowledge.

An orthotopic nude mouse model is required and has the potential to improve RFA research by imitating the in vivo environment. This aspect is important as the invasiveness and metastatic potential of HCC can be investigated under improved conditions compared with subcutaneous xenografts in nude mice $(18,19)$. However, the use of this orthotopic model for RFA is limited by the high mortality rate, as heat from the RFA needle electrode kills the tumor cells, but also damages the normal liver parenchyma that surrounds the tumor. In addition, restricting the application of the orthotopic model is required due to the highly complex surgical technique involved to maintain a high tumor formation rate in the animal liver, thus experienced laboratory technicians are essential. The orthotopic insufficient RFA model designed in the present study resolved the two aforementioned difficulties. The HCC nude mouse model (LCI-D20) was initially established 20 years ago, and has a reliable and stable tumor formation rate of $100 \%$ following orthotopic inoculation (12). Minimizing mortality is a vital step in model establishment. The third week (19-21 days) following xenograft inoculation was identified as the optimum RFA intervention time, as the xenograft tumor reached $\sim 0.5 \mathrm{~cm}$ in diameter. It is easier to achieve complete necrosis in smaller tumors during RFA. By contrast, bigger tumors require more time to reach the ideal state of insufficient RFA, leading to thermal damage to the surrounding normal liver parenchyma and death. Open abdominal rather than percutaneous RFA was selected in the present study, as the latter approach burns the skin at the puncture point and causes necrosis, which can cause death in a number of mice, as aforementioned. However, the open approach carries an increased risk of infection, but this is easily controlled by experienced operators. In addition, with the open approach, the xenograft tumor is observed directly without the requirement of ultrasonography, as the tumor protrudes from the liver surface. Thus, the insufficient RFA procedure is easier 

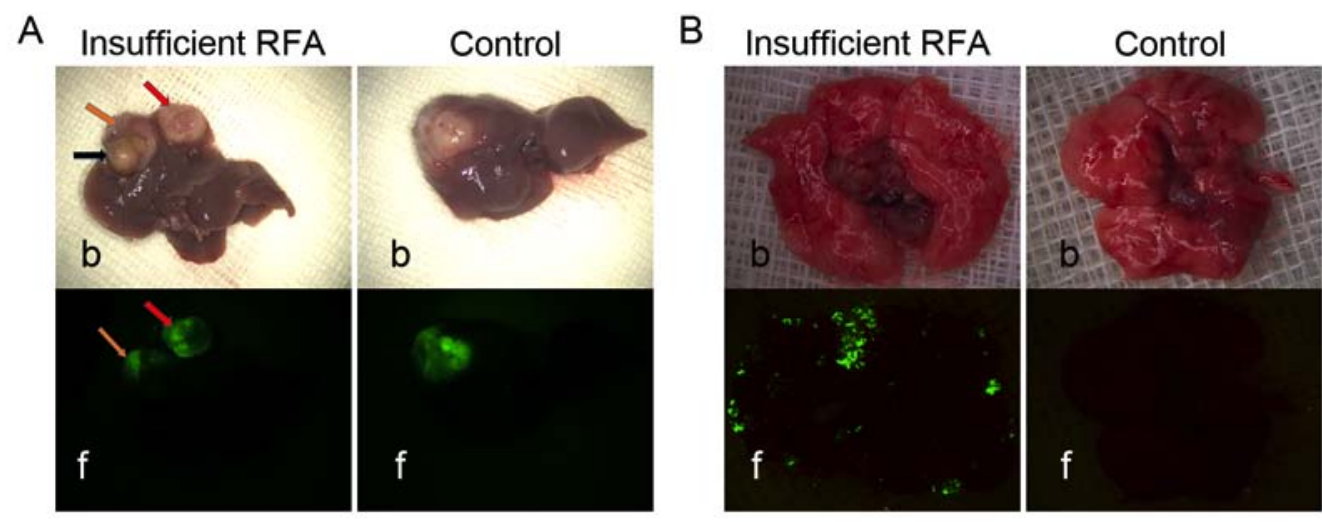

C

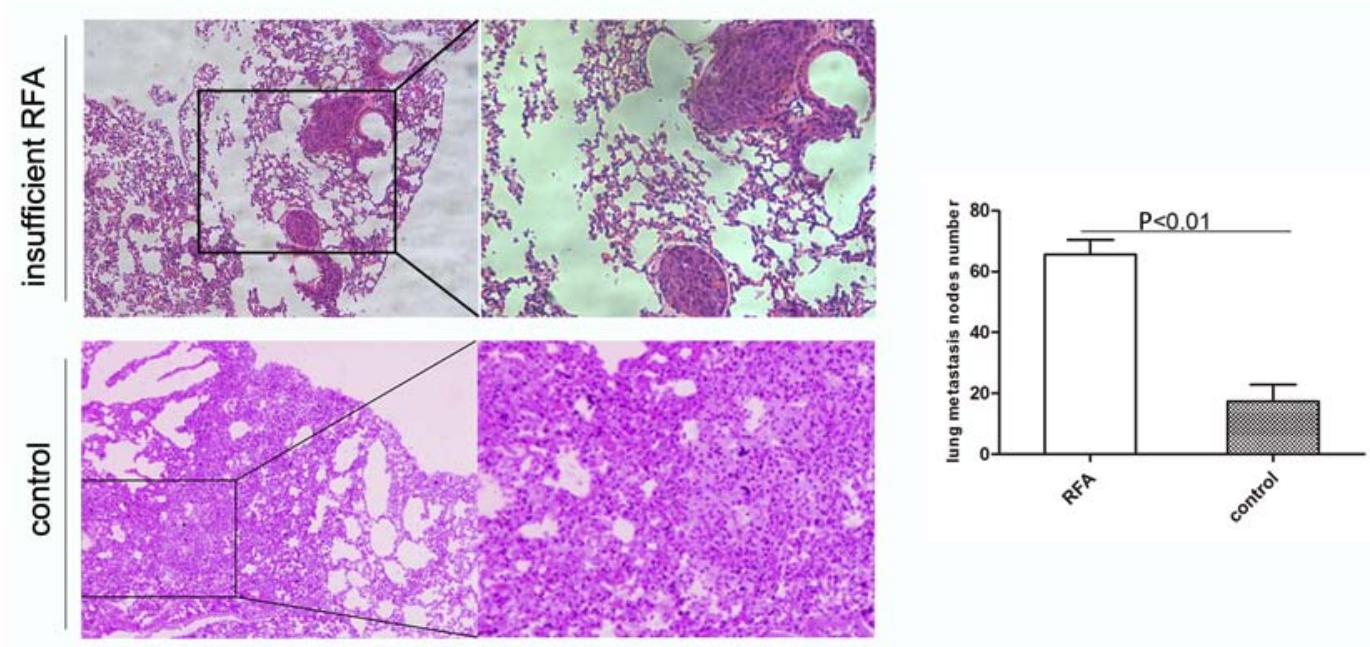

Figure 4. Insufficient RFA promotes invasiveness and distant metastasis. (A) Tissue labeled with the red arrow indicates IHM following insufficient RFA intervention, the orange arrow indicates residual tumor, while the black arrow indicates necrotic tissue following RFA. No IHM was observed under fluorescence microscopy in the tumor from the control group. (B) Lung metastasis in the insufficient RFA group was assessed by fluorescence microscopy. The green fluorescent areas represent metastatic nodules. The number of lung metastases in the insufficient RFA group (left image) was higher compared with that in the control group (right image). (C) Lung tissues were sectioned serially and stained with hematoxylin and eosin. There were significantly more nodes of lung metastases in the insufficient RFA group compared with the control group. P<0.01; Magnification, $\mathrm{x} 200$. b, bright field; f, fluorescence; IHM, intrahepatic metastasis; RFA, radiofrequency ablation.

to perform. A series of preliminary experiments as part of the present study, the parameters for RFA were, including the preset temperature, power and duration time. The RFA duration was the critical factor; for example, if the duration time was $>30 \mathrm{sec}$, the majority of nude mice suffered complete tumor necrosis and death. The parameters chosen in the present study avoided complete xenograft tumor necrosis and guaranteed residual cancer.

The present study was designed to establish an orthotropic nude mouse model and evaluate the invasiveness and metastasis of residual HCC following insufficient RFA. The model established in the present study is considered to be the first orthotopic nude mouse model to investigate insufficient RFA. The results demonstrated the significant influence of insufficient RFA on tumor growth and the invasiveness of HCC. The results revealed that insufficient RFA reduced xenograft tumor volume and prolonged the survival time of the nude mice. The residual tumor demonstrated more invasiveness and metastatic behavior in the insufficient RFA-treated mice, and insufficient RFA was followed by increased numbers of intrahepatic and lung metastatic nodules. The results from the present study are consistent with previous studies that revealed that insufficient RFA facilitated rapid progression of the residual tumor $(16,20,21)$. It has been reported that angiogenesis induced by hypoxia-inducible factor-1 $\alpha$ /vascular endothelial growth factor (VEGF)-A following hyperthermia may serve an important role in the rapid growth of the residual tumor following incomplete RFA (22). Ke et al (16) indicated that incomplete RFA facilitated the rapid progression of residual hepatic VX2 carcinoma by inducing the overexpression of several molecular factors, including proliferating cell nuclear antigen, matrix metalloproteinase-9, VEGF, hepatocyte growth factor and interleukin-6.

In conclusion, establishment of an insufficient RFA orthotopic nude mouse model facilitates research into the biological behavior of residual tumors and its underlying mechanisms. The results of the present study, utilizing this novel nude mouse model, shed novel insights on the pro-metastatic effects of insufficient RFA of HCC. Further preclinical research is further required to clarify the exact mechanism responsible for the metastasis-enhancing potential of residual tumors. 


\section{Acknowledgements}

Not applicable.

\section{Funding}

The present study was supported by the National Natural Science Foundation of China (grant no. 81372314).

\section{Availability of data and materials}

The datasets used and/or analyzed during the present study are available from the corresponding author on reasonable request.

\section{Authors' contributions}

NZ, HZ and YD performed the experiments. LW participated in the study concept and design. All authors read and approved the final manuscript.

\section{Ethics approval and consent to participate}

All animal protocols were approved by the Ethical Committee on Animal Experiments of Animal Care Committee of Fudan University (Shanghai, China).

\section{Patient consent for publication}

Not applicable.

\section{Competing interests}

The authors declare that they have no competing interests.

\section{References}

1. Siegel RL, Miller KD and Jemal A: Cancer Statistics, 2017. CA Cancer J Clin 67: 7-30, 2017.

2. Dutta R and Mahato RI: Recent advances in hepatocellular carcinoma therapy. Pharmacol Ther 173: 106-117, 2017.

3. Kawaguchi Y, Honda G, Endo I, Cherqui D and Kokudo N: Current technical issues for surgery of primary liver cancer. Liver Cancer 6: 51-58, 2016.

4. Lau WY and Lai EC: The current role of radiofrequency ablation in the management of hepatocellular carcinoma: A systematic review. Ann Surg 249: 20-25, 2009.

5. Shiina S, Tateishi R, Arano T, Uchino K, Enooku K, Nakagawa H, Asaoka Y, Sato T, Masuzaki R, Kondo Y, et al: Radiofrequency ablation for hepatocellular carcinoma: 10-year outcome and prognostic factors. Am J Gastroenterol 107: 569-578, 2012.

6. Feng K, Yan J, Li X, Xia F, Ma K, Wang S, Bie P and Dong J: A randomized controlled trial of radiofrequency ablation and surgical resection in the treatment of small hepatocellular carcinoma. J Hepatol 57: 794-802, 2012.

7. Park W, Chung YH, Kim JA, Jin YJ, Lee D, Shim JH, Lee D, Kim KM, Lim YS, Lee HC, et al: Recurrences of hepatocellular carcinoma following complete remission by transarterial chemoembolization or radiofrequency therapy: Focused on the recurrence patterns. Hepatol Res 43: 1304-1312, 2013.
8. Lam VW, Ng KK, Chok KS, Cheung TT, Yuen J, Tung H, Tso WK, Fan ST and Poon RT: Incomplete ablation after radiofrequency ablation of hepatocellular carcinoma: Analysis of risk factors and prognostic factors. Ann Surg Oncol 15: 782-790, 2008.

9. Zhang L, Yin X, Gan YH, Zhang BH, Zhang JB, Chen Y, Xie XY, Ge NL, Wang YH, Ye SL and Ren ZG: Radiofrequency ablation following first-line transarterial chemoembolization for patients with unresectable hepatocellular carcinoma beyond the Milan criteria. BMC Gastroenterol 14: 11, 2014.

10. Yang BW, Liang Y, Xia JL, Sun HC, Wang L, Zhang JB, Tang ZY, Liu KD, Chen J, Xue Q, et al: Biological characteristics of fluorescent protein-expressing human hepatocellular carcinoma xenograft model in nude mice. Eur J Gastroenterol Hepatol 20: 1077-1084, 2008

11. Wang L, Tang ZY, Qin LX, Wu XF, Sun HC, Xue Q and Ye SL: High-dose and long-term therapy with interferon-alfa inhibits tumor growth and recurrence in nude mice bearing human hepatocellular carcinoma xenografts with high metastatic potential. Hepatology 32: 43-48, 2000.

12. Sun FX, Tang ZY, Lui KD, Ye SL, Xue Q, Gao DM and Ma ZC: Establishment of a metastatic model of human hepatocellular carcinoma in nude mice via orthotopic implantation of histologically intact tissues. Int J Cancer 66: 239-243, 1996.

13. Bu W, Tang ZY, Sun FX, Ye SL, Liu KD, Xue Q, Chen J and Gao DM: Effects of matrix metalloproteinase inhibitor BB-94 on liver cancer growth and metastasis in a patient-like orthotopic model LCI-D20. Hepatogastroenterology 45: 1056-1061, 1998.

14. Kong J, Kong L, Kong J, Ke S, Gao J, Ding X, Zheng L, Sun $\mathrm{H}$ and Sun W: After insufficient radiofrequency ablation, tumor-associated endothelial cells exhibit enhanced angiogenesis and promote invasiveness of residual hepatocellular carcinoma. J Transl Med 10: 230, 2012.

15. Xu M, Xie XH, Xie XY, Xu ZF, Liu GJ, Zheng YL, Huang GL, Wang W, Zheng SG and Lü MD: Sorafenib suppresses the rapid progress of hepatocellular carcinoma after insufficient radiofrequency ablation therapy: An experiment in vivo. Acta Radiol 54: 199-204, 2013.

16. Ke S, Ding XM, Kong J, Gao J, Wang SH, Cheng Y and Sun WB: Low temperature of radiofrequency ablation at the target sites can facilitate rapid progression of residual hepatic VX2 carcinoma. J Transl Med 8: 73, 2010.

17. Nakagawa H, Mizukoshi E, Iida N, Terashima T, Kitahara M, Marukawa Y, Kitamura K, Nakamoto Y, Hiroishi K, Imawari M and Kaneko S: In vivo immunological antitumor effect of OK-432-stimulated dendritic cell transfer after radiofrequency ablation. Cancer Immunol Immunother 63: 347-356, 2014.

18. Massazza G, Tomasoni A, Lucchini V, Allavena P, Erba E, Colombo N, Mantovani A, D'Incalci M, Mangioni C and Giavazzi R: Intraperitoneal and subcutaneous xenografts of human ovarian carcinoma in nude mice and their potential in experimental therapy. Int J Cancer 44: 494-500, 1989.

19. Niederberger M, DeLozier-Blanchet CD, Hedinger CE and Walt H: Differences between subcutaneous and intraperitoneal forms of three human testicular teratocarcinomas in nude mice. Cancer 61: 1571-1578, 1988.

20. Kasugai H, Osaki Y, Oka H, Kudo M and Seki T; Osaka Liver Cancer Study Group: Severe complications of radiofrequency ablation therapy for hepatocellular carcinoma: An analysis of 3,891 ablations in 2,614 patients. Oncology 72 (Suppl 1): S72-S75, 2007.

21. Koda M, Murawaki Y, Hirooka Y, Kitamoto M, Ono M, Sakaeda H, Joko K, Sato S, Tamaki K, Yamasaki T, et al: Complications of radiofrequency ablation for hepatocellular carcinoma in a multicenter study: An analysis of 16346 treated nodules in 13283 patients. Hepatol Res 42: 1058-1064, 2012.

22. Kong J, Kong J, Pan B, Ke S, Dong S, Li X, Zhou A, Zheng L and Sun WB: Insufficient radiofrequency ablation promotes angiogenesis of residual hepatocellular carcinoma via HIF-1 $\alpha /$ VEGFA. PLoS One 7: e37266, 2012. 